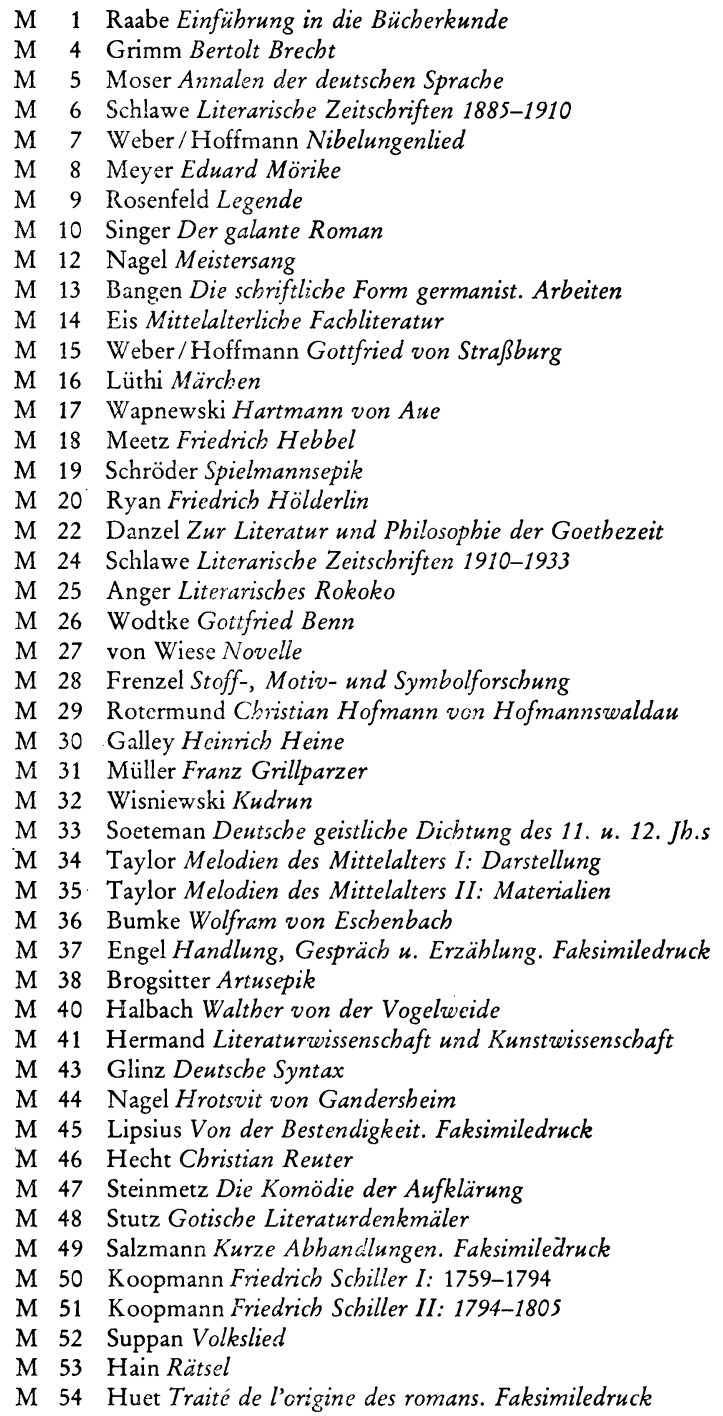




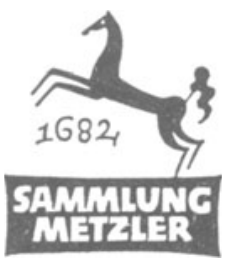

REALIEN ZUR LITERATUR

ABT. D:

LITERATURGESCHICHTE 
FRANK-RUTGER HAUSMANN

\section{François Rabelais}


Für Denise

CIP-Kurztitelaufnahme der Deutschen Bibliothek

Hausmann, Frank-Rutger:

François Rabelais / Frank-Rutger Hausmann. -

Stuttgart: Metzler, 1979.

(Sammlung Metzler; M 176: Abt. D,

Literaturgeschichte)

ISBN 978-3-476-10176-1

ISBN 978-3-476-10176-1

ISBN 978-3-476-03882-1 (eBook)

DOI 10.1007/978-3-476-03882-1

M 176

(C) 1979 Springer-Verlag GmbH Deutschland

Ursprünglich erschienen bei J. B. Metzlersche Verlagsbuchhandlung und Carl Ernst Poeschel Verlag GmbH in Stuttgart 1979 
Vorwort ................. VII

1. Rabelais und seine Zeit . . . . . . . . . . . . 1

1.1. Die politischen Verhältnisse . . . . . . . . 1

1.2. Rabelais' Leben und Werk . . . . . . . . . . . . 4

2. Rabelais-Rezeption und Stand der Rabelais-Forschung . . . 7

2.1. Vorbemerkung . . . . . . . . . . . . 7

2.2. Das Urteil der Zeitgenossen . . . . . . . . . . . . . 7

2.3. Kommentatoren . . . . . . . . . . . . . . . . . 9

2.4. Ubersetzer . . . . . . . . . . . . . . . . . . . . 12

2.5. Lexikographen . . . . . . . . . . . . . . . . . 15

2.6. Biographen . . . . . . . . . . . . . 15

2.7. Literaturkritiker . . . . . . . . . . . . 17

2.8. Nachahmer . . . . . . . . . . . . . . . 24

3. Die Einheit der Pentalogie . . . . . . . . . . . 27

3.1. Bisherige Deutungsversuche . . . . . . . . . 27

3.2. Die Genesis der Pentalogie; der >Pantagruel . . . . . 28

3.3. Der $>$ Gargantuar . . . . . . . . . . . . . . . . . 30

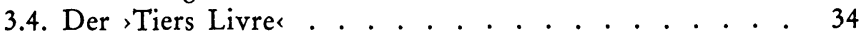

3.5. Der $\backslash$ Quart Livres. . . . . . . . . . . . . . . . 37

3.6. Der $>$ Cinquiesme Livrer . . . . . . . . . . . 40

4. Rabelais' Arbeitsweise . . . . . . . . . . . . . . . . 42

4.1. Rabelais und die Chroniques gargantuines< . . . . . . 42

4.2. Die Authentizität des $>$ Cinquiesme Livre`. . . . . . . 44

4.3. Die Prologe zu den einzelnen Büchern . . . . . . . . 49

4.4. Der Begriff des Pantagruelismus . . . . . . . . . . . 52

4.5. Der $>$ Gargantuar-Prolog . . . . . . . . . . . . . . 56

5. Quellen und Einflüsse im Werk Rabelais' . . . . . . . . . 60

5.1. Allgemeine Übersicht . . . . . . . . . . . . 60

5.2. Die Ritterromane . . . . . . . . . . . . . . . . . 62

5.3. Die Autoren der italienischen Renaissance . . . . . . 64

5.4. Der Einfluß der Antike . . . . . . . . . . . . . 67

5.5. Die Kenntnis anderer Wissenszweige . . . . . . . 71

6. Rabelais' Verhältnis zum Christentum .. . . . . . . . . 73

6.1. Vorüberlegungen . . . . . . . . . . . . . . . 73

6.2. Die religiöse Situation Frankreichs am Anfang des 16. Jhdts. . . . . . . . . . . . . . . . . . . . . 74

6.3. Rabelais und der orthodoxe Katholizismus . . . . . . 76

6.4. Rabelais und Luther, der Reformkatholizismus und Calvin . . . . . . . . . . . . . . 78

6.5. Rabelais und Erasmus . . . . . . . . . . . . . . . 82

7. Rabelais' politische Ideenwelt . . . . . . . . . . . . . 88

7.1. $>$ Religion, estat politicq et vie oeconomiquer . . . . . 88

7.2. Rabelais und die Monarchie .......... 90 
7.3. Außenpolitik . . . . . . . . . . . . . . . 93

7.4. Innenpolitik und soziale Verhältnisse . . . . . . . . 94

7.5. Literarische Quellen seines politischen Denkens . . . . 99

8. Komik, Erzähltechnik und sprachliche Neuerungen . . . 103

8.1. Forschungsstand und Selbstaussagen Rabelais' . . . . . 103

8.2. Komik und Komiktheorien . . . . . . . . . . . . . 106

8.3. Komische Verfahrensweisen im Werk Rabelais' . . . . 108

8.3.1. Gigantismus . . . . . . . . . . . . . 108

8.3.2. Karneval . . . . . . . . . . . . . . . . . 109

8.3.3. Wunschtraum, Vision, Alp . . . . . . . 110

8.3.4. Phantastische Reisen . . . . . . . . . . . . . 111

8.3.5. Parodie . . . . . . . . . . . . . . . . . 113

8.3.6. Satire und Porträttechnik . . . . . . . . . . 115

8.3.7. Sprachauffassung . . . . . . . . . . . . . . 118

8.3.8. Wortkomik . . . . . . . . . . . . . 122

Zeittafel zu Werk und Leben Rabelais' . . . . . . . . . . . 128

Auswahlbibliographie . . . . . . . . . . . . 134

Namensverzeichnis . . . . . . . . . . . . 153 


\section{VORWORT}

Deutsche Einführungen in Werk und Leben Rabelais' sind nicht gerade zahlreich; eine entsprechende selbständige Studie gibt es im Augenblick unseres Wissens nicht. Das trotz seiner Einseitigkeit im großen und ganzen verdienstvolle Büchlein von Heintze (B. 213) ist vergriffen und war in der Bundesrepublik stets nur schwer erhältlich. Es versteht sich zudem (S. 211) als fortschrittliche demokratische Bemühung um Rabelais und klammert die Ergebnisse der jüngeren Rabelais-Forschung aus. Buck (B. 141) und Stackelberg (B. 359) bieten zwar gute Einführungen in Rabelais' Werk, aber ihre Darlegungen sind Teile von Sammelbänden und der Natur der Sache nach relativ knapp. Für Militz (B. 277), ebenfalls Teil eines Sammelbandes, gilt ähnliches wie für Heintze. Substantiell ist Bucks Einleitung in den Rabelais-Band der Reihe "Wege der Forschung " (B. 53), aber sie stellt nur einen knappen Forschungsbericht dar. Distelbarth (B. 179) beschränkt sich auf den erzähltechnischen Aspekt, Radtke (B. 306) auf den Stil; beide Arbeiten sind im übrigen im Handel nicht erhältliche Dissertationen.

So soll das vorliegende Büchlein, das sich in erster Linie als Realienband versteht, einem Leser nicht nur Informationen über den Autor Rabelais und sein Werk vermitteln, sondern auch die Schwierigkeiten aufzeigen, die eine Rabelais-Lektüre bietet. Zugleich will es die wichtigsten Ergebnisse der RabelaisForschung mitteilen. Die Kenntnis des Inhalts der fünf Bücher Gargantua et Pantagruel (im folgenden die »Pentalogie" genannt), wird dabei stillschweigend vorausgesetzt; eilige Leser können Einzelheiten in Hatzfelds Inhaltsangabe (B. 211) nachschlagen; auch auf die einschlägigen deutschen Übersetzungen sei verwiesen (B. 74-79).

Rabelais wird im Original nach der von Jourda erstellten zweibändigen Garnier-Ausgabe (B. 64) zitiert, die einzelnen Bücher der Pentalogie im fortlaufenden Text mit G. (=Gargantua), $P$. (= Pantagruel), T. L. (= Tiers Livre), Q. L. (= Quart Livre), C. L. (= Cinquiesme Livre), im Zitat mit I, II, III, IV, $\mathrm{V}$ plus Kapitelangabe und Seitenzahl der Garnier-Ausgabe abgekürzt. Wir bieten als Lesehilfe, wenn nicht ausdrücklich anders vermerkt, die deutsche Übersetzung von Widmer und Horst (B. 79), die dem heutigen Sprachstand angemessener ist als die in ihrer Zeit bahnbrechende Übersetzung von Regis (B. $74 ; 75)$. Auch hier sei auf gleichwertige andere Ausgaben ver- 
wiesen (Pléiade, B. 62; TLF, B. 67-69; 71; 72) oder die wohlfeile Intégrale (B. 66), die zudem den Vorteil einer neufranzösischen Ubersetzung neben dem mittelfranzösischen Original bietet. Die im Lauf unserer Darlegungen zitierten lateinischen Autoren (Erasmus, Calvin, Du Puy-Herbault u. a.) werden sogleich in eigener deutscher Übersetzung geboten.

Als Abschluß eines jeden Kapitels findet sich eine thematische Bibliographie, die auf eine fortlaufend numerierte Gesamtbibliographie am Ende des Bandes verweist (B.), und zwar mit dem Autornamen und der Nummer der Bibliographie. Dementsprechend wird auch im Lauf der Untersuchung zitiert. Diese Bibliographie ist eine Auswahlbibliographie, die nur wichtige und aktuelle Titel nennt, die fast alle in die Untersuchung einbezogen wurden.

Zum Schluß noch eine methodische Vorbemerkung: Die Rabelais-Forschung hat in den letzten Jahren erneut einen großen Aufschwung genommen. Seit dem letzten umfassenden Forschungsbericht von Schrader (B. 87) sind laut Klapp ca. 800 neue Titel zu Rabelais erschienen. Es ist unmöglich, sie alle zu rezipieren oder gar miteinander zu versöhnen, zumal die Rabelais-Forschung in zwei divergierende Richtungen zerfällt. Defaux (B. 164) spricht in seiner lesenswerten Einleitung von einer »querelle des anciens et des modernes«. Wer sich mit Rabelais beschäftigt, muß in diesem Streit Stellung beziehen. Die »anciens«, vertreten durch Forscher wie Defaux, Françon, Krailsheimer, Larmat, Marichal, Saulnier, Screech u. a. gehen davon aus, daß man Rabelais' Werk nicht verstehen könne, "sans la replacer dans son temps«. Die »modernes « wie Beaujour, Glauser, Keller, Paris, Tetel u. a. wollen sich nicht mit historisch-eruditem Ballast befrachten, sondern heben in erster Linie auf den erzähltechnischen und komischen Eigenwert der Pentalogie ab.

Wir bekennen uns eher zum Lager der »anciens«, sind jedoch der Meinung, daß sich beide Sehweisen miteinander ohne weiteres vereinbaren lassen, da eine allzu einseitige Betrachtung dem Kunstwollen Rabelais' nicht gerecht würde. Schon Spitzer (B. 356) war der Auffassung, daß das Reale bei Rabelais Sprungbrett des Irrealen sei. Auch eine marxistische Deutung, wie sie Lefebvre (B. 251) bereits 1956 vorschlug, die Forscher wie Beaujour (B. 124) und Paris (B. 290) fortführen, scheint uns eher der Wunschvorstellung ihrer Verfechter als der Realität, d.h. dem Werk und Denken Rabelais', zu entspringen. Diese Exegeten sehen in Rabelais den Zerstörer des Mittelal- 
ters, "qui vise à étendre les ravages à toutes les catégories de la culture " (Paris, B. 290, 213) und eine neue Zukunft vom Dynamismus der Wissenschaften und der Aktivierung der Bürger und Bauern erhoffe.

Rabelais ist jedoch ein eher konservativer Autor, der gegen Habsburg, Luther, das Rittertum, den Kolonialismus, Partikularismus, Kapitalismus usw. eingestellt ist und einer bäuerlichpatriarchalischen Sozialordnung zuneigt. Dem widerspricht auch nicht die Tatsache, daß Rabelais Humanist ist bzw. im Lauf seines Lebens und damit auch im Lauf seines schriftstellerischen Schaffens seinen Standpunkt modifiziert hat. Der Humanismus geht von einer Wiedererweckung der Antike mit allen ihren Lebensbereichen aus, ist seiner Tendenz nach also restaurativ. Die mustergültigen Erkenntnisse der Antike und Spätantike, soweit sie Philosophie, Recht, Literatur, Kunst, Architektur, Staatswesen usw. betreffen, sind nach Meinung der Humanisten im Mittelalter entweder vergessen oder doch falsch verstanden worden. Es gilt, sie von diesen Verkrustungen zu befreien und sie in ihrer ursprünglichen Gestalt wiedererstehen zu lassen. Dieses Gedankengut hat sich Rabelais teilweise zu eigen gemacht. Seine spezifische Leistung ist es, es in ein volkssprachliches Werk integriert zu haben, das den Meisterwerken der Antike wie auch den theologisch-philosophischen Traktaten seiner Zeit ebenbürtig an die Seite treten kann.

Freiburg, im Juli 1978

F.-R. H. 